\title{
Kernos
}

Revue internationale et pluridisciplinaire de religion grecque antique

$30 \mid 2017$

Varia

\section{Le dieu fou. Essai sur les origines de Śiva et Dionysos}

Philippe Swennen

\section{(2) OpenEdition}

\section{Journals}

Édition électronique

URL : http://journals.openedition.org/kernos/2515

DOI : 10.4000/kernos.2515

ISSN : 2034-7871

\section{Éditeur}

Centre international d'étude de la religion grecque antique

\section{Édition imprimée}

Date de publication : 1 octobre 2017

Pagination : 326-329

ISSN : 0776-3824

\section{Référence électronique}

Philippe Swennen, «Le dieu fou. Essai sur les origines de Śiva et Dionysos », Kernos [En ligne], 30

2017, mis en ligne le 01 octobre 2017, consulté le 24 septembre 2020. URL : http://

journals.openedition.org/kernos/2515; DOI : https://doi.org/10.4000/kernos.2515

Ce document a été généré automatiquement le 24 septembre 2020.

Kernos 


\title{
Le dieu fou. Essai sur les origines de Śiva et Dionysos
}

\author{
Philippe Swennen
}

\section{RÉFÉRENCE}

Bernard SERGENT, Le dieu fou. Essai sur les origines de Śiva et Dionysos, Paris, Les Belles

Lettres, 2016. 1 vol. 14,8 × 21,5 cm, 442 p. (Vérité des mythes). ISBN : 978-2-251-38571-6.

1 La collection «Vérité des mythes » des Belles Lettres accueille l'ouvrage par lequel Bernard Sergent rouvre un dossier classique mais difficile, celui de la description et de l'élucidation des accointances qui, selon différents comparatistes, lient le Dionysos de la Grèce classique au Śiva du monde hindou. L'A. reprend le débat avec une intention fermement énoncée : démontrer le caractère hérité, donc l'identité originelle commune, de ces deux divinités. L'ouvrage comporte une «Introduction. La religion souterraine » (9-17), que suivent les douze chapitres de la démonstration; I. "Śiva n'est pas Rudra » (19-25) ; II. "Mort et renaissance » (27-67) ; III. " Manifestations » (69-128); IV. «Les forces de vie»(129-220); V. «Un cycle de fêtes» (221-237); VI. « Aspect physique » (239-266) ; VII. «Verticalité » (267-291) ; VIII. « Les noms du dieu » (293-304) ; IX. «Initiation » (305-329) ; X. « Les arts » (331-343) ; XI. « Mythes » (345-376) ; XII. «Autres parallélismes» (377-400). Ce dernier chapitre consiste à identifier dans d'autres domaines de l'aire indo-européenne que le grec et l'indien des divinités présentant des traits suggérant une équivalence à Dionysos et Śiva. L'ouvrage prend fin par une «Conclusion » (401-405) que suivent des "Références » réparties entre les «Abréviations » (407-412) et la rubrique « Ouvrages et articles cités » (412437). La « Table des matières » occupe les pages 439 à 442.

2 Les talents de plume de l'A. sont reconnus de longue date. Comme d'habitude, la lecture de l'ouvrage est plaisante, absorbante même et, sous les réserves techniques qui seront développées ci-dessous, facilitée par le fait que l'A. ne perd jamais le fil de son exposé. L'indianiste se heurte ponctuellement à des tournures un peu abruptes, comme lorsque 
Yama est qualifié de « divinité de la Mort » (p. 40), là où il vaudrait mieux dire que cette figure mythologique, que le formulaire ancien ne désigne jamais comme un dieu, est souverain des enfers pour avoir été le premier à faire l'expérience de la mort. Il note aussi certaines distractions dans la translittération des mots sanskrits, par exemple le Brāman㷂a de la page 11, ou encore le récurrent kamā des pages 349 à 357 . On discourrait en vain de ce qu'est une bibliographie exhaustive dans un essai de mythologie comparée. Ce point sera ponctuellement rouvert plus bas. On se bornera à remarquer que la bibliographie de B.S. est très personnelle, mêlant d'incontestables travaux de référence (Detienne, Doniger, Dumézil, etc.) à d'autres, beaucoup moins sûrs (Daniélou, Evola...). La lecture de l'ouvrage suscite par ailleurs l'impression confuse que la rédaction du travail a commencé il y a longtemps déjà, puis a fait l'objet d'un toilettage tardif et un peu superficiel, en ce compris au niveau de la bibliographie. Ainsi par exemple l'attention est-elle attirée par le fait que la monographie bien connue de Wendy Doniger O'Flaherty est qualifiée de « récente» (p. 21). En sciences humaines, la notion est évidemment relative, mais l'ouvrage date tout de même de 1973, sa traduction française de 1993.

3 L'entreprise conduite par l'A. se heurte d'emblée à un écueil considérable, évident pour tout indianiste: le caractère apparemment secondaire de Śiva. Ce dernier est totalement inconnu dans la strate archaïque de la poésie religieuse sanskrite, à savoir les hymnes du Rigvéda. Sa figure est textuellement constituée dans la Śvetāśvatara Upanișad, donc dans la strate la plus tardive de la littérature canonique védique. Elle est alors liée d'emblée à la pratique ascétique et au yoga. Elle semble ensuite s'affirmer de manière constante à travers les siècles et les productions littéraires successives, jusqu'à devenir la principale bénéficiaire des dévotions relevant de la bhakti classique. L'érudition occidentale valide habituellement l'information indienne selon laquelle cette immense et complexe figure divine équivaudrait originellement à l'inquiétant dieu védique Rudra, colérique et destructeur, complètement réinterprété pour susciter l'archétype de l'ascète investi de la gestion des transformations ravageuses accompagnant inévitablement les changements de cycle temporel. Il est permis d'affirmer que ce schéma diachronique bénéficie davantage d'un consensus mou que d'une authentique démonstration probante (chapitre I).

B.S., que ne peut satisfaire cette proclamation du caractère secondaire de Śiva, peine toutefois à construire un cadre plus solide dans les pages de son «introduction». Il recourt en fait à une idée aussi ancienne qu'invérifiable, celle selon laquelle la religion des hymnes du Rigvéda serait superficielle en ce qu'elle n'aurait concerné que les élites de la société. D'autres croyances souterraines, dont l'existence serait suggérée par les découvertes archéologiques, typiquement celles illustrant un culte de la déesse mère, auraient finalement réussi à imposer leurs vues, suscitant donc ce résultat paradoxal que les nouveautés seraient en fait pour l'essentiel l'apparition à la lumière du jour de croyances préhistoriques jusqu'alors refoulées. À la fois parce qu'elles sont issues de la population ordinaire et parce qu'elles donnent une importance essentielle aux thématiques relatives à la fécondité, ces croyances relèvent de la troisième fonction du schéma dumézilien. C'est dans sa conclusion que B.S. plaidera qu'une sorte de compromis entre brahmanes orthodoxes et menu peuple contrecarrera l'émergence du bouddhisme inhérente au règne d'Aśoka par la promotion, à partir du $\mathrm{II}^{\mathrm{e}}$ siècle avant l'ère commune, d'un hindouisme que renouvelait une sorte d'inversion hiérarchique du panthéon plaçant à l'avant-plan les divinités populaires traditionnelles. 
5 La vraisemblance du schéma est d'autant moins sûre qu'aucun volet historique ne l'étaye. Or, la période de transformation postulée par l'A., et dont le volet bouddhique est évidemment certain, est aussi celle durant laquelle les mondes grec et indien entretiendront divers contacts, notamment diplomatiques. Il est aujourd'hui très difficile de dire ce qu'il en résulta, mais au moins certains essaient-ils, qui ne peuvent se prévaloir d'être pris en compte par B.S. Il est connu depuis déjà longtemps que les témoins grecs rebaptisent conventionnellement Śiva " Dionysos ", dieu des montagnes qu'ils opposent à Vișn臨u-Héraklès, dieu des plaines. La réévaluation de ces données a été accomplie par Guillaume Ducoeur, «Interpretatio, relectures et confusions chez les auteurs gréco-romains: le cas du Dionysos indien", Mythos, Rivista di Storia delle Religioni, supplément 2 (2011), p.143-158, dont l'absence de la bibliographie de l'ouvrage est une faiblesse objective. Certes, l'influence de données poétiques indiennes sur un auteur grec est probablement indémontrable, mais il n'est tout de même pas indifférent qu'une œuvre littéraire soit antérieure ou postérieure à la période des échanges culturels lorsqu'il s'agit d'invoquer les traces de résurgence d'un patrimoine hérité. L'objection devait être formulée, compte tenu notamment de la place occupée dans l'ouvrage par les Dionysiaques de Nonnos de Panopolis.

6 On rappellera par ailleurs que l'hypothèse du substrat religieux caché va le plus souvent de pair avec la définition par pétition de principe du système religieux traditionnel de la vieille civilisation de l'Indus antérieure à l'implantation des populations qui produiront les hymnes védiques en recourant au vieux matériel formulaire qu'elles se transmettent par tradition orale : selon cette vue, le dieu ascète existait certes avant les Védas, mais dans la population autochtone antérieure à l'installation des locuteurs du sanskrit, et c'est par syncrétisme qu'il finit par émerger à nouveau.

7 Implicitement, B.S. repousse cet argument pour y substituer une sorte d'entassement de données dont la masse doit bien finir par constituer un faisceau de présomption contraignant. Il le fait avec l'érudition, l'énergie et l'intuition qui lui sont habituelles, mais celles-ci ne parviennent toutefois pas à emporter l'adhésion du lecteur à la thèse de l'A. En effet, diverses difficultés méthodologiques s'élèvent, que la lecture de l'ouvrage ne dissipe pas.

8 La présentation du dossier repose donc sur l'énumération de nombreux traits que B.S. perçoit comme comparables et communs aux deux dieux. La première objection qui s'impose à la lecture de l'ouvrage est que le matériel textuel est assez faiblement mis en valeur. Quand un texte est cité, c'est en traduction. La plupart du temps, les références des passages utilisés sont simplement mentionnées en note de bas de page. Il arrive aussi que la source soit indirecte, reprenant des informations relatées par des auteurs parfois anciens. Ainsi le lecteur qui se sent de taille à consulter lui-même les textes grecs et sanskrits sur base desquels B.S. bâtit son plaidoyer doit-il prévoir sur son bureau la même bibliothèque que l'A. Ce n'est évidemment pas un détail. Outre le fait que certaines des sources très fréquemment invoquées sont d'un usage délicat, comme c'est typiquement le cas de la littérature orphique ou des Purānas, le lecteur ne peut pratiquement jamais vérifier par lui-même la nature du parallélisme entre les matériels lexicaux comparés. C'est une faiblesse, pour au moins deux raisons.

9 La première est que la parenté lexicale justifierait l'indifférence pour l'hypothèse indusienne en établissant fermement la réalité de l'origine indo-européenne. La seconde, bien plus grave, est qu'il serait ainsi éventuellement possible de circonscrire 
un vocabulaire spécifique à ces deux dieux, ce qui serait le meilleur argument actuellement disponible pour tenter de démontrer leur racine commune. Dans l'état présent des connaissances, le mépris de B.S. à l'égard de cette méthode ne peut que heurter. Le paroxysme de cette attitude est peut-être atteint aux pages 131 à 139 de l'ouvrage. À en croire l'A., la présence du serpent parmi les nombreux signes exprimant les «forces de vie » fait partie des traits communs unissant Dionysos et Śiva. Le moins que l'on puisse dire est que ce n'est pourtant pas leur exclusivité. Tant en Grèce qu'en Inde, une multitude de récits mythiques comportent la présence de serpents ou de dragons. Le thème du meurtre du serpent est aujourd'hui perçu comme l'archétype du mythème hérité identifiable au caractère récurrent d'une formule indo-européenne, par exemple *ég ${ }^{\text {wh }}$ ent ó óghim, utilisée et réinterprétée dans de nombreuses langues plus ou moins anciennes. Cette utilisation de la théorie de la formule permettant de cerner un formulaire préhistorique est l'avancée accomplie notamment par Calvert Watkins, dont l'ouvrage de synthèse, How to kill a dragon, paru en 1995, est très étonnamment absent de la bibliographie de B.S. Du coup, sous le sous-chapitre "Serpent» du chapitre IV, l'A. rassemble des faits disparates dont le seul point commun est la présence, explicite ou non, de serpents, sans qu'il en résulte de solide unité. Dans une version orphique de la naissance de Zagreus, Zeus prend la forme d'un serpent pour s'unir à Perséphone, ce qui permet à B.S. de dire que Dionysos est né du serpent (p.131). Quelques pages plus loin, nous apprenons que, à la fin des Dionysiaques, le même dieu combat des Géants et des troupes de serpents (p. 135). Certes, mais quel lien logique et structurant peut-on établir entre la filiation et l'antagonisme ? Dans sa danse cosmique, Śiva danse en ayant un cobra enroulé autour du bras, ce qui évoque une image des bacchantes (p. 137). Est-ce assez pour identifier les sectatrices de Dionysos au dieu indien? Dans l'épopée indienne, lors du barattage de la mer de lait, un poison émane de la masse lactée. Śiva l'avale et en garde la gorge bleue, ce qui le rendrait seul capable de guérir le venin des serpents (p. 138). Mais ce qui compte ici, est-ce le serpent où l'inhibition temporaire par Śiva de forces destructrices? Du reste, nul a-t-il jamais vu Dionysos faire montre de la même aptitude guérisseuse en absorbant quelque venin que ce soit? La conclusion de B.S. est la suivante : « Les éléments par lesquels Dionysos est en relation avec le serpent couvrent presque exactement ceux par lesquels Śiva est lui aussi lié au serpent. » Faisons l'effort de l'admettre, fût-ce de mauvaise grâce. Ces éléments, quel domaine circonscrivent-ils? Quel trait du portrait du dieu hérité désignent-ils? À quel acte de sa geste spécifique correspondent-ils ? Enfin et surtout, quelle relation au reste du panthéon et quelle fonction spécifique permettent-ils d'identifier?

Les exemples de ce type truffent littéralement l'ouvrage, au point d'en être le véritable ressort, et c'est la raison pour laquelle l'esprit refuse de se rendre devant cette espèce de déversement massif d'informations hétérogènes. Une quantité énorme de données dont la vérification systématique prendrait des semaines entières ne conduit en apparence qu'à l'entassement de tout un ensemble de paramètres dont il est impossible de mesurer la spécificité. De combien de dieux du monde indo-européen ancien peut-on dire qu'ils ont plusieurs noms, peuvent changer d'apparence, sont liés au taureau ou au lion, font l'objet de cérémonies printanières ou automnales, sont ornés de guirlandes et mentionnés par des symboles représentant la verticalité ? Surtout, de combien de dieux étrangers au monde indo-européen pourrait-on en dire tout autant?

11 Le défaut de départ est sans doute que le cadre du paysage hérité est trop faiblement caractérisé. Certes, B.S. reste fidèle à la tripartition fonctionnelle, mais cette clé 
analytique reste inefficace. La lecture de l'ouvrage suscite pourtant d'importantes questions. L'étude des portraits de ces deux dieux complexes, dont la nature paradoxale semble finalement le vrai point commun, suscite l'hypothèse selon laquelle, en Inde comme en Grèce, un espace était réservé à un dieu dont la mission était précisément d'exprimer les limites, les lacunes ou les inconséquences de cet ordre harmonieux, qu'elles se soient trouvées inscrites dans les réalités de la nature physique ou dans les consciences humaines. Est-ce bien ce que représentent Śiva et Dionysos? Il faudrait l'établir fermement avant de tâcher de dégager une origine commune de cette éventuelle accointance.

\section{AUTEURS}

\section{PHILIPPE SWENNEN}

(Université de Liège) 